\title{
Carcinoma in situ de seno
}

\author{
Fernando Perry*
}

\begin{abstract}
RESUMEN: En el Instituto Nacional de Cancerología, una revisión de 10 años se encuentra alrededor de 20 casos de Carcinoma In Situ;
\end{abstract} todos los tumores eran palpables, y el diagnóstico fue casual.

Esta experiencia tan frustrante ha motivado la revisión de la literatura y el estudio de este cuadro patológico desde el punto de vista de su historia natural, temas que se presentan en este trabajo.

El avance técnico en los aparatos de mamografía y su uso cada vez más amplio, permite esperar un aumento en la frecuencia de este diagnóstico, por lo cual se considera conveniente que el cuerpo médico se familiarice con esta patología que surgió recientemente. En alguna medida este artículo trata de ayudar a su conocimiento.

PALABRAS CLAVES: Carcinoma ductal in situ, carcinoma lobulillar in situ.

\section{Introducción}

El cáncer de seno es una enfermedad que despierta cada vez más atención, no sólo en la comunidad médica sino en el grupo de población femenina por explicables razones. Las inherentes a la mujer como la amenaza a su vida por su conocida y difundida alta mortalidad, así como la deformidad estética y la disfunción sexual son las más conocidas. Preocupa también su creciente incidencia, inicialmente en los países desarrollados pero ahora detectada también en los países en vía de desarrollo, probablemente por la repetición en éstos de los factores de riesgo para la enfermedad que ya se han detectado en aquéllos.

En Colombia, país considerado como de incidencia baja de la enfermedad se nota un incremento estadístico a través de los informes del Plan Nacional de Cáncer, así es como en este momento se sitúa entre las tres primeras causas de muerte en el grupo de población de 45 a 65 años de edad.

El Instituto Nacional de Cancerología atiende anualmente alrededor de 550 casos nuevos de Cáncer de Seno de los cuales el $80 \%$ están clasificados en estados III y IV, es decir casos avanzados. Visto de otra manera hay $1 \%$ de cánceres in situ y queda un $20 \%$ a ser repartido entre estados I y II. La conclusión es que en este momento no existe un plan de detección de Cáncer de Seno coherente que permita disminuir la mencionada alta mortalidad de la enfermedad.

Por otra parte, los países desarrollados tienen ya en marcha planes de detección precoz cuyos reportes mues-

\footnotetext{
* Cirujano Oncólogo. Docente del Servicio de Cirugía General y
} seno. Instituto Nacional de Cancerología. tran una disminución en la mortalidad por Cáncer de Seno, directamente proporcional a sus índices de diagnóstico temprano.

En este trabajo se pretende revisar el tema de Cáncer de Seno in situ como una forma de promover su conocimiento, así sea en este momento teórico. También se trata de que se tome conciencia de un cambio de actitud hacia el uso de los parámetros de la mamografía para una búsqueda más activa de la enfermedad incipiente. Estos son resumidos de los que están en uso en este momento en EEUU en el cuadro 1. Se recuerdan también los factores de riesgo más importantes en el cuadro 2.

Se trata de que el médico no espere a que le llegue el estado avanzado sino que tome una parte activa en el diagnóstico de lesiones incipientes mediante el uso racional de la mamografía.

Además de la discusión de las generalidades de cada cuadro histológico se menciona el método a seguir para el diagnóstico radiológico, la toma de la muestra por cirugía y su procesamiento. Se propone la unificación de los términos en patología haciendo hincapié en las características de cada lesión las cuales son muy importantes para entender los tratamientos propuestos.

\section{Cáncer de seno in situ}

\section{Carcinoma ductal in situ}

Carcinoma lobulillar in situ

\section{Carcinoma lobulillar in situ}

\section{Definición}

Se origina en las células epiteliales que recubren la luz de los acinos mamarios. Estas tienen un tamaño un 
poco mayor que el de las normales. Las mitosis son poco frecuentes. Progresivamente van llenando la luz de los acinos hasta obliterarla y pueden extenderse hasta los conductos.

Esta extensión a la luz ductal se ve con más frecuencia en mujeres ancianas cuyas glándulas muestran atrofia de los acinos.

Fue descrita inicialmente por James Ewing quien la denominó "Proliferación atípica de células acinosas, alteraciones precancerosas y proliferación atípica en un segmento del conducto". Foote y Stewart en 1941 consolidaron esta noción patológica como una enfermedad aparte y la denominaron "Carcinoma Lobulillar in situ una forma de cáncer mamario".

Este nombre está hoy en discusión debido a que Haagensen un estudioso del tema, consideró que tal denominación le daba un carácter de falsa agresividad que podía condicionar conductas muy radicales.

Por esta razón propuso el término de "Neoplasia Lobulillar in Situ" buscando con esto acentuar el carácter que él consideraba más importante de marcador de riesgo para cáncer de seno invasivo y no el de lesión preinvasiva que fatalmente terminará en cáncer invasivo. En realidad lo que este autor busca es que se entienda que la mujer portadora de esta patología tiene un riesgo aumentado para cáncer invasivo pero también que la mayoría de estas pacientes no terminaran desarrollándolo.

Esta lesión por supuesto no tiene un carácter infiltrativo y está confinada al acino sin ultrapasar la membrana basal. Es de destacar que no existe un criterio morfológico que permita predecir cuál evolucionará al desarrollo de un tumor invasivo.

\section{Diagnóstico mamográfico}

Tradicionalmente, se ha considerado que el CLis no tiene un cuadro radiológico típico. En realidad,destinado a ser un diagnóstico casual antes del advenimiento de la mamografía moderna este punto había permanecido muy impreciso. Con el uso de la mamografía en programas de tamizaje se han identificado algunas imágenes que con alguna frecuencia corresponden a NLis como son distorsiones o retracciones del parénquima mamario o microcalcificaciones redondeadas uniformes y agrupadas. Más adelante es posible que se identifique un patrón imagenológico más preciso.

\section{Diagnóstico diferencial patológico}

El principal es el de la hiperplasia lobulillar atípica. Las características de las dos son muy parecidas, siendo la diferencia bastante sutil en cuestión de cantidad de células anormales o su disposición. Por esto es deseable un patólogo con experiencia en este campo para mayor seguridad. Este también dará mayores seguridades al conocer el minucioso método de corte y examen de cada pieza para descartar focos de microinvasión.

Otros diagnósticos diferenciales son la papilomatosis cuando tiene un patrón sólido o el Ca ductal in situ de tipo cribiforme aunque la presencia de células anaplásicas ayudan para su diagnóstico.

\section{Incidencia}

Por ser ésta una lesión oculta hasta hace poco, cuyo diagnóstico casual ha sido errático, por su cuadro histológico que apenas ahora comienza a ser aceptado de manera más universal, su incidencia se considera tentativa. La serie de Haagensen, reportada antes de la mamografía moderna y realizada en material patológico obtenido por diagnósticos diferentes muestra como el $3.8 \%$ de todas las lesiones epiteliales benignas. En la serie de Wheeler es el $0.8 \%$ y para Andersenen 3.299 lesiones benignas es el $1.5 \%$.

Parece claro que estas series reportan porcentajes puramente incidentales. El uso cada vez más amplio de la mamografía en la cual se han identificado algunas imágenes sugestivas lo que ha llevado a un número mayor de biopsias así como el estudio más cuidadoso de los especímenes quirúrgicos ha cambiado las cifras hasta encontrar un reporte de un $8 \%$ en especímenes de biopsia. Parece que habrá que esperar todavía para encontrar la verdadera incidencia.

\section{Edad}

La mayoría de autores reportan un promedio de edad de 45 años siendo que, en la serie de Haagensen el $88 \%$ estaba en edad premenopáusica.

\section{Multicentricidad bilateralidad}

La multicentricidad definida como áreas de carcinoma en otros sitios de la glándula se reporta entre el 60 al $90 \%$.

La bilateralidad puede variar entre el 25 y el $70 \%$.

Se explica la diferencia tan grande en estas cifras por los diferentes tipos de selección de casos, tipo de muestreo, número muy bajo de casos, criterios de diagnóstico patológico y el muestreo de la misma mama o de la opuesta.

Las cifras son altas en relación a otros carcinomas y en todo caso indican que todos los acinos están sometidos a un proceso similar y a estímulos iguales. Así pues debemos pensar que esta enfermedad es de los dos senos.

Es de aclarar que la definición de multifocalidad y multicentricidad no es usada universalmente por todos los autores con el mismo sentido.

\section{Historia natural}

La historia natural del CARCINOMA LOBULILLAR IN SITU, es poco conocida en buena parte por ser una lesión de diagnóstico incidental. La época de post mamografía moderna con su correspondiente diagnóstico histológico más frecuente, seguramente arrojará más luces sobre ésta.

Conocemos que esta lesión tiene receptores de estrógenos positivos más frecuentemente que su correspondiente CA DUCTAL IN SITU; también sabemos que su detección es muy baja en mujeres postmenopáusicas, las cuales paradójicamente soportan un mayor número de biopsias que las mujeres en edad premenopáusica.

Lo anterior ha hecho pensar a algunos autores, que al llegar a la edad de la menopausia algunas de estas 
lesiones pueden sufrir regresión espontánea, además de que se presume también que esta lesión tiene algún grado de hormodependencia. (Fryberg et al Surg Gynecol Obstet 164: 265-301, 1987).

EL CARCINOMA LOBULILLAR IN SITU, se localiza más frecuentemente en la región retroareolar o en el cuadrante superoexterno en donde está la mayor cantidad de tejido mamario. Norris (Norris $\mathrm{Hj}$ et al, anal Quant Cytol Hystol 10: 1-9. 1987). En su serie reporta esta localización hasta en un $90 \%$ y otros autores como Hagensen hasta el $87 \%$.

Esta lesión se asocia frecuentemente con otro tipo de Carcinomas como es el Ductal, que cuando es de tipo tubular tiene un pronóstico mejor.

Cuando se estudia esta asociación, se encuentra que el Carcinoma Ductal Invasivo, supera en amplia proporción al Lobulillar Invasivo en época post menstrual. Como se sabe que los dos In Situ coexisten con frecuencia, se supone que la predominancia del Ductal Invasivo post menopáusico, se debe a su mayor agresividad, ya que se convierte en invasivo con más frecuencia, así como su velocidad de crecimiento es mayor. Por otra parte, también el lobulillar In Situ contribuiría a esta diferencia, si admitimos que al llegar a la menopausia, bien disminuye su velocidad de crecimiento o bien presenta regresión.

\section{Carcinoma lobulillar in situ como marcador de riesgo para cáncer invasivo posterior}

Este punto es particularmente destacado por todos los autores, en donde se considera que radica la mayor utilidad de esta entidad patológica. Aunque existen los dos conceptos, aquél que dice que esta enfermedad termina fatalmente como Cáncer Invasivo, y aquél que dice que su diagnóstico implica un mayor riesgo para cáncer invasivo, aceptando que una buena proporción de estas mujeres no presentarán la enfermedad invasiva; la tendencia actual es más a considerarlo como un marcador de riesgo.

En una serie del Memorial Sloan Ketterin (Hutter Foote), en 49 pacientes con CARCINOMA LOBULILLAR IN SITU, seguimiento de 4-27 años, ocurrió carcinoma invasivo en $14 / 49$ pacientes, o sea $29 \%$ y en $17 /$ 89 mamas $(19 \%)$.

En la serie de la Universidad de Pennsylvania (Wheeler), en 35 pacientes con seguimiento promedio de 15 años, ocurrió CARCINOMA en 6/59 mamas (10.2\%) y en 6/35 pacientes (17\%). La serie del hospital Sundby de Copenhague (Andersen) en 52 pacientes con un seguimiento promedio de 15 años, encontró Carcinoma en 18/ 98 mamas (18\%), y en $15 / 52$ pacientes (28\%).

La serie del Memorial (Rosen) que actualiza Ladefoott con un seguimiento medio de 24 años, mostró la ocurrencia de Carcinoma en el $36.9 \%$ de las pacientes. La serie del Mount Sinai (Toker-Gouldberg) en 178 pacientes mostró una incidencia acumulativa de Carcinoma Invasivo a los 20 años, en el seno homolateral de $19 \%$ y en la mama opuesta de $14 \%$.

En la serie de Haagensen se encontró una ocurrencia de Carcinoma Invasivo de 7 veces más de lo esperado para las tasas de incidencia calculadas en la región de Columbia.

En una revisión de 515 casos de literatura, se encontraron 37 pacientes fallecidas por Carcinoma, es decir, un $7 \%$ de mortalidad con una variación en la literatura de 4 a $19 \%$. Estos casos de Carcinoma Invasivo, se pueden asociar con metástasis axilares hasta en un $20-50 \%$ de acuerdo con la serie consultada.

También se reporta asociación con Carcinoma Invasivo Simultáneo, que según Rosen es de un $4 \%$ en su serie. Una revisión de la literatura muestra un promedio del $7 \%$, siendo la incidencia para cada una de las mamas del $5 \%$. De estas lesiones invasivas simultáneas el $65 \%$ corresponden a CARCINOMA DUCTAL.

\section{Tratamiento}

El tratamiento del Carcinoma Lobulillar In Situ, puede ser muy variado, dependiendo del punto de vista que adopte el médico tratante. Si se acepta el criterio de Haagensen dándole valor sobretodo a su valor predictivo, la conducta puede ser observación, teniendo en cuenta que la mamografía no muestre una lesión extensa o bilateral o multifocal.

El extremo opuesto lo constituye la consideración de que ésta es una enfermedad que afecta a los dos senos en una alta proporción de casos, por lo cual, siguiendo este criterio se debería tratar bilateralmente. Adicionalmente está su asociación en cáncer invasivo simultáneo hasta en un 7 por ciento o de desarrollo ulterior hasta en un $25 \%$ con mortalidad del $7 \%$.

Para seguir la conducta de observación, se debe tener claro que la apariencia mamográfica no muestre otras lesiones o una extensión muy grande, así como el hecho de que la paciente debe ser una paciente de fácil seguimiento, así como que ésta estará en capacidad de cumplir la rutina de seguimiento clínico y mamográfico, desde el punto de vista económico. Esto implica autoexamen mensual cuidadoso que debe ser enseñado por el propio médico, examen clínico con el especialista cada 4 meses o semestral y mamografía semestral en los primeros dos años y después anual.

En el caso de que se quiera ser intervencionista existen varias opciones. Una cuadrantectomía más muestreo axilar de la base, es para algunos conducta aceptable que permite estudiar el parénquima mamario, en donde está implantada la lesión, lo que ayuda a identificar el seno como de bajo o de alto riesgo, así como el muestreo ganglionar permite una mayor seguridad en cuanto a que no se haya subdiagnosticado una lesión invasiva.

Para otros, dada su multicentricidad se considera más aceptable la mastectomía simple ampliada, que es aquella que incluye los ganglios de la base axilar en la disección. De nuevo, este tratamiento permite un control locoregional de la enfermedad muy bueno, así como detectar un eventual foco de CARCINOMA INVASIVO, en cuyo caso los estudios de los ganglios de la base permiten un estudio más completo de la lesión invasiva. Otros autores preconizan con mucha lógica la mastectomía simple ampliada bilateral basados en que el riesgo del 
carcinoma invasivo existe para los dos senos, por lo cual el tratamiento debe incluirlos a los dos. En la actualidad esta conducta, en el caso en que se desee hacer tratamiento quirúrgico, tiende a generalizarse, dada la facilidad de reconstrucción simultánea que se ofrece con las nuevas técnicas de cirugía plástica.

No se considera un tratamiento adecuado la mastectomía subcutánea por los múltiples reportes de CARCINOMA INVASIVO después de este procedimiento.

\section{Carcinoma ductal in situ}

\section{Definición}

Es aquél que muestra en la histología células ductales con aspecto de malignidad, que no invaden el estroma, es decir, no invaden la unión entre el tejido epitelial y el tejido conectivo.

Hay la tendencia actual hacia la idea de que no es una sola entidad sino que incluye varios tipos de lesiones. Para entender esto de manera práctica, un autor (Connory) la define como una proliferación de células potencialmente malignas en el interior de la luz del sistema ductal. Schwartz por su parte lo considera como un T. maligno, pero que no invade todavía. Estas lesiones ocupan un lugar intermedio entre las hiperplasias y el Carcinoma Invasor.

\section{Clasificación patológica}

En la era anterior a la mamografía moderna el Carcinoma Ductal In Situ se consideró de una manera simplista como una lesión única. En esa época el diagnóstico era incidental, las pacientes con este diagnóstico muy escasas y la actitud médica en general de desinterés por el tema.

En la actualidad con el uso de la mamografía moderna, que, dentro de los parámetros establecidos, tiende a generalizarse cada vez más, se diagnostica cada vez más en los países desarrollados. Esto ha llevado a profundizar en su estudio y la correlación clínico-patológica ha llevado a la clasificación actual en la cual el cuadro morfológico microscópico, tiene su correspondiente historia natural y pronóstico, con las consiguientes implicaciones terapéuticas.

Hay 5 subtipos ya aceptados:

Tipo comedo

Tipo No comedo: Cribiforme sin necrosis

Micropapilar

Sólido

Cribiforme con necrosis

\section{Papilar intraquístico (Carter)}

El carácter multifocal y multicentral que acompaña esta lesión ha llevado a varias interpretaciones, un esquema tridimensional tratando de explicar que cuando se hacen múltiples cortes, en realidad se están viendo varios focos de una lesión in situ que se ha extendido por entre los conductos según Andersen (Acta Oncológica, 27, 739-743. 1988); se clasifican desde el punto de vista tridimensional en 3 tipos:
Microfocal: Presencia de lesión en una o varias unidades lobulillares, sin alteraciones del estroma y con un diámetro no mayor de $5.0 \mathrm{~mm}$.

Difuso: Lesiones mayores de 5. Mm.

Formador de tumor: en el cual no se encuentran glándulas ni conductos con distribución normal. Estroma fibrótico o de tipo inflamatorio y mide más de $5.0 \mathrm{~mm}$.

Vamos a describir histológicamente cada uno de los subtipos de Carcinoma Ductal IN Situ:

\section{Carcinoma In Situ no comedosiano}

Tiene varios patronos como cribiforme sin necrosis, micropapilar y sólido. Rara vez se ven puros en cuyo caso el más frecuente es el cribiforme, con mayor frecuencia tiene patrones histológicos que se mezclan.

\section{Cribiforme con necrosis}

Tiene un patrón histológico intermedio entre el comedo y el no comedo, según; clasificación basada en los conceptos de Peage, Page y Lagos (Cáncer 50: 1309-1314, 1982).

\section{Carcinoma Ductal In Situ tipo Comedo}

A menudo tiene un halo de fibrosis alrededor de la lesión lo que explica en parte la formación de masa palpable que se ve a veces. Contiene material similar al del comedón común, de ahí su nombre, que corresponde a material necrótico. Puede tener desde tamaño mínimo en los casos detectados por mamografía hasta lesiones muy extensas que pueden abarcar un porcentaje importante de la glándula mamaria, de estas características ha derivado el término de componente ductal extenso que implica una extensión amplia de la lesión vista por mamografía, generalmente más de un cuadrante.

\section{Carcinoma papilar intraquístico}

Descrito por Karter y colaboradores (Karter D, OrrOrsl Merino M.J. Cáncer 52: 14-19, 1983) caracterizado por la presencia de pedículos fibrovasculares rodeados por el epitelio proliferativo, debe ser diferenciado de la variante micropapilar comentada antes.

El término intraquístico, se usa para describir no invasión; cuando no está asociado a componente de Carcinoma In Situ. en áreas adyacentes, se considera que la resección local amplia es curativa, aún más si se tiene en cuenta que estas lesiones por lo general se presentan en pacientes de edad avanzada.

Aunque esta clasificación tiene utilidad, no es usada de manera uniforme por los diferentes patólogos, por lo cual hoy en día se recomiendan a éstos en presencia de una de estas lesiones, se debe describir ésta con todo detalle histológico, así como su tamaño. Esto porque sabemos que hay diferencia entre una lesión microscópica, con escasas atipias o sin ellas, contrapuesta a una lesión grande y con múltiples atipias, en cuanto a pronóstico se refiere.

\section{Diagnóstico}

La posibilidad de detección de carcinoma ductal in situ se ha incrementado enormemente con el uso de la mamografía. El diagnóstico se hace por la capacidad de 
estas lesiones de depositar calcio en los conductos y conductillos. Estos depósitos se asocian con la lesión central, con restos celulares, que a su vez se asocian con necrosis. Estos fenómenos se ven más frecuentemente en el tipo comedo y también menos frecuentes en el cribiforme. Sin embargo, cualquier lesión ductal in situ puede producir microcalcificaciones.

La composición de las microcalcificaciones corresponde a depósito de hidroxiapatita o fosfato tricálcico con algunos otros componentes.

\section{Factores pronósticos}

Existen factores pronósticos, que un patólogo puede realizar en su propio laboratorio, como son el tamaño de la lesión, la subclasificación histopatológica, grado de diferenciación celular, reacción fibrosa perilesional.

El tamaño es factor pronóstico ya que se sabe que las lesiones tienen menos posibilidad de microinvasión en cuanto más pequeñas sean. Se ha establecido que una lesión de más de $2.5 \mathrm{cms}$ de diámetro se asocia más frecuentemente a microinvasión.

En cuanto mayor sea la extensión de la enfermedad más frecuentemente hay focos de carcinoma invasivo.

La clasificación por subtipos nos indica que el comedocarcinoma es la lesión más agresiva, la que presenta en las series indicadas más falla local, más focos de microinvasión y más frecuencia de metástasis axilares.

Generalmente, la atipia marcada se asocia con peor pronóstico así como con el tipo comedo o con lesiones con mayor reacción fibrosa perilesional.

Los métodos complementarios de esta clasificación pronóstica histológica no se analizan en nuestro país, pero de todas maneras se comentan. Ellos son importantes porque en conjunto con los factores histológicos ayudan en las decisiones terapéuticas en cada caso individual.

La cuantificación del material genético de las células tumorales, que cuando es normal se llama diploide, y cuando es anormal aneuploide, proporciona información de la siguiente manera. Los casos de aneuploida tienen más posibilidades de asociarse con microinvasión. (Carpenter R./ et al Br J Surge 74: 905-906, 1987). (De Poteertt Cr. et al Histopathology 11: 1307-1319, 1987).

El estudio del oncogen Erb - B - 2 (NEU) (Vandde Viujer et al N. Eng. J. Med - 319: 1239-1245, 1988) reveló que un $41 \%$ de 45 casos de esta serie tenían sobreexpresión de este oncogen.

Llama la atención en este trabajo que todos ellos, corresponden al tipo comedo, y son de peor pronóstico.

Carpenter en su estudio del contenido genético celular tumoral, encuentra que un $90 \%$ de los casos con microinvasión son Aneuploides.

Estudios con marcación con Timidina que indican la cinética del ciclo celular, es decir, el porcentaje de células que están en proceso de síntesis de DNA han encontrado que los casos de Comedocarcinoma tiene un índice de más de el doble de el de las otras lesiones.

Como se ve el estudio de estos factores pronósticos va sacando en claro aquellos casos con peor pronóstico, con las implicaciones terapéuticas obvias.

\section{Historia natural}

Los datos disponibles en la literatura son escasos, ya que estas pacientes en su mayoría han sido tratadas con mastectomía y por lo cual no se ha estudiado su evolución después de un diagnóstico; algunas escasas series de pacientes seguidas después de biopsia con diagnóstico de carcinoma ductal in situ, muestran:

En 25 pacientes con seguimiento de 16 años promedio, una incidencia del $28 \%$ de carcinoma invasivo (Page et al Cáncer 49: 751-758, 1982).

(Betsil et al Jama 239: 1863-1867, 1978) en 10 pacientes con seguimiento de 21 años promedio, muestra una incidencia de 7 casos de cáncer invasivo para un porcentaje del $70 \%$. En estas series se calcula la probabilidad de cáncer invasivo después de biopsia de 25 a $50 \%$.

De esto se calcula que hubo una mortalidad por cáncer del $10 \%$ y el tiempo promedio para la aparición de lesión invasiva es de 10 años después del diagnóstico. Las lesiones invasivas se observan en su mayoría en el mismo cuadrante en que se hizo el diagnóstico inicial. Las series comentadas, tenían en su mayoría pacientes con lesiones grandes, ya que son de la era anterior a la mamografía moderna.

En la actualidad, aunque hay una amplia variación en los datos, se considera que el carcinoma ductal in situ detectado por mamografía, es decir, la lesión pequeña no es tan agresiva, puede tener un curso indolente, si bien, si se deja progresar sin tratamiento, tiene una probabilidad mucho mayor que la de un carcinoma lobulillar de terminar en una lesión invasiva.

\section{Diagnóstico radiológico}

Las dos lesiones comentadas tienen la tendencia a ser diagnosticadas cada vez más de manera temprana. El uso de los programas de tamizaje demostrará cada vez con mayor frecuencia estas lesiones. Es fundamental pues que se familiarice a todos los médicos que están en este tema con los métodos diagnósticos.

Estas lesiones tienen signos mamográficos sugestivos de ellas. En el caso del Carcinoma Lobulillar in situ se acepta hoy que cuando las microcalcificaciones son puntiformes y redondeadas o cuando hay distorsión del parénquima puede corresponder a esta leșión. Estos signos son imprecisos y se espera con el tiempo mejorar su efectividad.

El caso del Carcinoma Ductal in situ es diferente por cuanto las señales son más sugestivas. Así las microcalcificaciones tradicionalmente irregulares, de formas bizarras, ramificadas siguiendo el patrón ductal de los conductos, agrupadas en un corto espacio, a veces de forma alargada o asociadas con masa.

En cualquiera de los dos casos una vez que se ha hecho el diagnóstico de sospecha de una lesión mamográfica debe procederse de la siguiente forma.

Como estas lesiones no son palpables debe pedirse la ayuda del radiólogo quien procede a marcarla con aguja especial, que puede ser de diferentes tipos, pero cuya característica es que tiene una punta en forma de arpón, para que quede inmovilizada. 
El paso siguiente es proceder a la extirpación quirúrgica bajo anestesia general de la lesión marcada (ver esquema). La pieza quirúrgica debe ser remitida a $\mathrm{Rx}$, para radiografía de manera que se compruebe que la extirpación se hizo correctamente. La Rx de la pieza puede hacerse seleccionándola en varios fragmentos para localizar en cual de ellos, están las microcalcificaciones sospechosas. Este fragmento se envía marcado al patólogo, quien además de revisar toda la pieza pondrá más énfasis en el segmento marcado.

En la era anterior a la mamografía moderna el carcinoma ductal in situ en EEUU, era menos del 5\% de todos los casos nuevos de cáncer de seno. En los 90, es el $20 \%$ de todos los casos nuevos, siendo, más del 70\% detectados por mamografía (Basset L, Radklin of NA, 30: 1,93105, Jang 92). El carcinoma ductal in situ puede presentar dos tipos:

El primero que se asocia con el tipo comedo, las microcalcificaciones se producen como consecuencia de depósitos de calcio, en sitios de necrosis central, del material celular intraductal. Su apariencia sigue el patrón ductal, alargada, con ramificaciones.

En el segundo tipo las microcalcificaciones se producen por estancamiento de las secreciones dentro de los espacios quísticos o en los intersticios de las proyecciones tumorales. Corresponden al tipo cribiforme y tienen una apariencia radiológica puntiforme con formas bizarras irregulares, variables en forma y tamaño.

Estas imágenes pueden estar mezcladas en la medida que los cuadros histológicos son mixtos.

La mamografía es el único método de detectar microcalcificaciones las cuales a su vez, son el signo más precoz de cáncer de seno incipiente. Ningún otro método puede lograr una detección tan temprana, por lo cual, TAC, R.N.M., ecografía y termografía no lo han podido reemplazar como método de tamizaje.

Para lograr la visualización de imágenes tan pequeñas de 1.0 ó $2 \mathrm{mms}$., es imprescindible que el examen mamográfico cumpla con los siguientes requisitos.

Debe contarse con un mamógrafo, es decir un equipo diseñado únicamente para este examen. Este mamógrafo utiliza una técnica de compresión del seno, componentes como el tubo de molibdeno, bajo kilovoltaje y placa de alto contraste con un procesador especial. Estos detalles técnicos no los cumple un aparato de Rx convencional por lo que se recomienda no hacer mamografía en éste.

\section{Comentarios}

Hay muchas opciones terapéuticas en estas dos entidades y la obligación del médico es informar ampliamente a cada paciente, para que ella tome parte activa en la escogencia.

Hay la tendencia a generalizar el tratamiento conservador, y dentro de las consideraciones ya dichas, en el aparte de tratamiento en el INC se está de acuerdo en realizarlo. Esto sin embargo, debe ser realizado siguiendo criterios estrictos, ya que la recidiva local y regional del tumor en estas pacientes, puede constituirse en una amenaza para su vida y en un verdadero desafío para el tratamiento. Las pacientes que recidivan, pueden ser tratadas con mastectomía simple de rescate. Para todo cancerólogo la cuestión del control locoregional de la enfermedad constituye preocupación importante porque es él quien generalmente debe enfrentar este problema, que puede ser extremamente difícil de tratar.

\section{Tratamiento}

El tratamiento es quirúrgico. En presencia de una lesión localizada con factores de buen pronóstico se acepta cirugía conservadora V-GR. Una tumorectomía amplia acompañada o no de muestreo axilar o vaciamiento axilar. Esto también es válido para el tipo no comedo. En el caso de comedo, o no comedo con componente intraductal extenso (que se diagnostica por mamografía por múltiples áreas sospechosas de microcalcificaciones diseminadas) y/o factores de mal pronóstico se recomienda mastectomía simple ampliada con reconstrucción inmediata o tardía. 